\title{
Exosomes Secreted by Mesenchymal Stem Cells Induce Immune Tolerance to Kidney Transplantation via Transporting IncRNA DANCR
}

\section{Xiaoqiang $\mathrm{Wu}$}

zhengzhou university people's hospital

\section{Zhiwei Wang}

zhengzhou university prople's hospital

Junpeng Wang

zhengzhou university people's hospital

Xiangyong Tian

zhengzhou university people's hospital

\section{Guanghui Cao}

zhengzhou university people's hospital

Yue Gu

zhengzhou university people's hospital

\section{Fengmin Shao}

zhengzhou university people's hospital

Tianzhong Yan ( $\nabla$ yantzhong666@163.com )

zhengzhou university people's hospital https://orcid.org/0000-0001-6146-0092

\section{Research Article}

Keywords: IncRNA DANCR, SIRT1, kidney transplantation, Treg cells, BMMSCs, exosomes

Posted Date: June 12th, 2021

DOI: https://doi.org/10.21203/rs.3.rs-585446/v1

License: (1) (1) This work is licensed under a Creative Commons Attribution 4.0 International License.

Read Full License 


\section{Abstract}

\section{Background}

Mesenchymal stem cells induce kidney transplant tolerance by increasing regulatory $\mathrm{T}$ (Treg) cells. Bone marrow mesenchymal stem cell exosomes (BMMSC-Ex) promote Treg cell differentiation. Long noncoding RNA differentiation antagonizing non-protein coding RNA (DANCR) is expressed in BMMSCs and can be encapsulated in exosomes. We aimed to explore the role of DANCR in BMMSC-Ex in immune tolerance after kidney transplantation and related mechanism.

Methods

The kidney transplantation model was established and levels of serum creatinine (SCr) were determined. Hematoxylin-eosin staining was conducted to detect the inflammation and immunohistochemistry was performed to detect the infiltration of $\mathrm{CD}^{+}{ }^{+} \mathrm{T}$ cells. Levels of IFN- $y$, IL-17 and IL-2 were examined by ELISA. Flow cytometry was conducted to determine Treg cells.

Results

In allograft group, the inflammatory response was severe, $\mathrm{CD} 4^{+} \mathrm{T}$ cell infiltration, $\mathrm{SCr}$ levels, and plasma rejection related factors were up-regulated, while injection of BMMSC-Ex reversed the results. BMMSC-Ex increased Treg cells in kidney transplantation mice. Interference with DANCR reversed the promoting effect of BMMSC-Ex on Treg cell differentiation. DANCR bound to SIRT1, promoted ubiquitination and accelerated its degradation. The injection of BMMSC-Ex (after interference with DANCR) promoted SIRT1 levels, inflammatory response, $\mathrm{CD} 4^{+} \mathrm{T}$ cell infiltration, $\mathrm{SCr}$ levels, and plasma rejection related factors' expression, while Treg cells were decreased.

Conclusion

LnCRNA DANCR in BMMSC-Ex promoted Treg cell differentiation and induced immune tolerance of kidney transplantation by down-regulating SIRT1 expression in $\mathrm{CD} 4^{+} \mathrm{T}$ cells.

\section{Introduction}

Kidney transplantation is a common treatment for chronic renal insufficiency at the endstage [1]. However, it still faces challenges such as long-term survival of receptors and adverse reactions to immunosuppressive agents, which can lead to an overall suppression of the immune system, increasing infection rates, and the incidence of tumors [2].

Regulatory T (Treg) cells are a subpopulation of T cells with independent functions, and forkhead box P3 (Foxp3) is the main surface marker [3]. Liao $T$ et al. found that induced Treg cells significantly weakened the histological graft injury and rejection, and significantly improved the survival of kidney allograft [4]. In addition, Mesenchymal stem cells (MSCs) has been reported to induce kidney transplantation tolerance 
by increasing the number of Treg cells [5]. Studies have shown that exosomes can delay kidney graft rejection and participate in immune tolerance of kidney transplantation [6-8]. However, the effect of MSCderived exosomes on immune tolerance of kidney transplantation has not been clearly reported. Besides, exosomes secreted by bone marrow mesenchymal stem cells (BMMSCs) promote differentiation of CD ${ }^{+}$ T cells into Treg cells $[9,10]$. We hypothesized that exosomes secreted by BMMSCs promote kidney transplantation immune tolerance by promoting $\mathrm{CD} 4^{+} \mathrm{T}$ cell differentiation into Treg cells.

As the main medium of information communication between MSCs and other cells, exosomes have been reported to realize the regulation function of MSC on target cells by encapsulating a variety of molecules including long non-coding RNA (IncRNA), microRNA (miRNA), and mRNA [11]. LncRNA differentiation antagonizing non-protein coding RNA (DANCR) is a tumor suppressor that plays a tumor-suppressive role in renal cell carcinoma [12]. In addition, DANCR is significantly decreased in acute kidney injury, and it can promote the viability and inhibits apoptosis of renal tubular epithelial cells treated with LPS [13]. Therefore, we suggested that DANCR plays a protective role in kidney injury. Furthermore, DANCR is expressed in BMMSCs and can be encapsulated in the exosomes [14, 15]. We hypothesized that BMMSCs might play a role in kidney transplantation by secreting exosomes encased with DANCR.

Sirtuin-1 (SIRT1) is an important target of immunotherapy, elimination of SIRT1 in T cells promotes the differentiation of $\mathrm{CD} 4^{+} \mathrm{T}$ cells into Treg cells and enhances Foxp3 levels $[16,17]$. In addition, inhibition of SIRT1 can improve the patient survival and the function of transplanted kidney [18].

Therefore, we hypothesized that IncRNA DANCR in BMMSCs exosomes promoted Treg cell differentiation and induced kidney transplantation immune tolerance by down-regulating the expression of SIRT1.

\section{Materials And Methods}

\section{BMMSCs collection and culture}

Femurs and tibias of 6-week-old C57BL/6 female mice were collected, washed with phosphate buffered solution (PBS) containing $2 \%$ fetal bovine serum (FBS), and inoculated in DMEM medium containing $2 \%$ FBS and penicillin/streptomycin. The adherent cells were removed $48 \mathrm{~h}$ later. After the adherent cells reached $90 \%$ fusion, the adherent cells were treated with trypsin [19]. Mice were raised in the Animal Center of Zhengzhou University. All animal procedures met the standards of the Institutional Animal Care and Use Committee of Zhengzhou University People's Hospital.

\section{BMMSCs exosomes (BMMSC-Ex) collection}

BMMSCs cells were cultured in depleted exosomal medium (cells were centrifuged at $100000 \mathrm{~g}$ ), the supernatant was collected and centrifuged at $300 \mathrm{~g}$ for $10 \mathrm{~min}, 3000 \mathrm{~g}$ for $10 \mathrm{~min}, 20,000 \mathrm{~g}$ for $30 \mathrm{~min}$, and $120,000 \mathrm{~g}$ for $70 \mathrm{~min}$. BMMSC-Ex were obtained after resuspended in PBS [20].

\section{Transmission electron microscopy (TEM)}


TEM was conducted to observe the morphology of BMMSC-Ex using a Tecnai ${ }^{\text {TM }}$ transmission electron microscope (FEl, USA). Before photography, BMMSC-Ex was suspended in $2.5 \%$ glutaraldehyde and loaded on the copper grids [19].

\section{Nanoparticles Tracking Analysis (NTA)}

The size of BMMSC-Ex was determined by NTA with Nanosight NS300 (Malvern, UK). Exosomes were resuspended by PBS, diluted, and injected into the sample chamber. The NTA software was used to capture and analyze the data.

\section{Western blot}

Proteins of BMMSC-Ex or CD4 ${ }^{+} \mathrm{T}$ cells were collected with RIPA Lysis Buffer (Beyotime, Shanghai), and the samples were quantified by Pierce ${ }^{T M}$ BCA Protein Assay Kit (Thermo Fisher Scientific, USA). The samples were administrated with SDS-PAGE followed by transferred to the polyvinylidene difluoride membrane (Millipore, Germany). The membrane was blocked and then incubated with primary antibodies against CD9 (ab92726, 1:2000), TSG101 (ab125011, 1:1000), CD63 (ab217345, 1:1000), SIRT1 (ab12193, 1:2000). Calnexin (ab22595, 1:1000) and $\beta$-actin (ab8227, 1:1000) overnight at $4^{\circ} \mathrm{C}$. The membranes were then incubated with Goat Anti-Rabbit IgG H\&L (HRP) for $2 \mathrm{~h}$. Finally, the blots were observed with BeyoECL Moon (Beyotime Biotechnology, Shanghai) [21].

\section{Animal model}

Mice were grouped into isograft control (BALB/c to BALB/c, $n=7$ ), allograft (C57BL/ 6 to $B A L B / c, n=7$ ), allograft + PBS (C57BL/6 to BALB/c, n=7), allograft + BMMSC-Ex (C57BL/ 6 to BALB/c, n=7). For the model of isogenic kidney transplantation, $B A L B / c$ mice were performed as donors and recipients. For the model of allograft kidney transplantation, $\mathrm{C} 57 \mathrm{BL} / 6$ mice were acted as donors and BALB/c mice were the recipients.

Mice were anaesthetized with pentobarbital sodium and ether, and subcutaneously injected with butorphanol (1 mg/kg) for analgesia. In donor mice, a median abdominal incision was made, and the left kidney was used as the donor kidney, the left kidney and blood vessels were exposed. The abdominal aorta and the inferior vena cava were dissociated, ligated, and blocked. After perfusion, the abdominal aorta, inferior vena cava and ureter were moved, and the left kidney and its associated blood vessels and ureter were stored in $4^{\circ} \mathrm{C}$ high osmotic citron salt adenine solution. In recipient mice, a median abdominal incision was made, and the left kidney was resected, the donor kidney was placed into the left lower abdomen. Then, $2 \mathrm{~mL}(50 \mathrm{U} / \mathrm{mL})$ of $4^{\circ} \mathrm{C}$ heparin saline was injected into the right peritoneum for systemic heparinized. The transplanted blood vessel was first anastomosed to the recipient aorta and then to the inferior vena cava. After the anastomosis, the blood supply was restored and the ureter was implanted into the bladder. The cold ischemia time was $60 \mathrm{~min}$ and the hot ischemia time was $20 \mathrm{~min}$. 
For allograft + PBS group, mice were infused with PBS through caudal vein 1 day before and 1 day after kidney transplantation. For allograft + BMMSC-Ex group, mice were infused with BMMSC-Ex $(10 \mu \mathrm{g}$, suspended in PBS) through caudal vein 1 day before and 1 day after kidney transplantation. All animal procedures met the standards of the Institutional Animal Care and Use Committee of Zhengzhou University People's Hospital [6].

\section{HE (hematoxylin-eosin) staining}

Six days after transplantation, the kidney tissues were collected, fixed with $10 \%$ formaldehyde, dehydrated with different concentrations of alcohol gradient, embedded in paraffin, and cut into $5 \mu \mathrm{m}$ slices. Tissues were then dewaxed with xylene, rehydrated with gradient alcohol and stained with HE (Beyotime Biotechnology, Shanghai). After gradient dehydration and transparency, the kidney tissues were sealed with neutral gum and observed under a light microscope (Olympus BX51, Japan).

\section{CD4 staining}

Six days after transplantation, the kidney tissues were collected, fixed with $4 \%$ paraformaldehyde, incubated with $0.3 \% \mathrm{H}_{2} \mathrm{O}_{2}$ for $30 \mathrm{~min}$, blocked with Tris-buffered saline containing $10 \%$ normal serum and $1 \%$ Bull Serum Albumin for $2 \mathrm{~h}$, and incubated overnight with Anti-CD4 antibody (ab183685, 1:200) at $4^{\circ} \mathrm{C}$. The next day, sections were washed and incubated with biotin-labeled secondary antibody at room temperature for $1 \mathrm{~h}$, following stained with 3,3'-diaminobenzidine for 10 min. Finally, the images were observed under a microscope (Zeiss, Germany) [22]

\section{Serum creatinine $(\mathrm{SCr})$ levels}

Blood samples were taken from mice and centrifuged at $1500 \mathrm{~g}$ for $15 \mathrm{~min}$. SCr levels were examined using an Indiko automatic biochemical analyzer (Thermo Fisher Scientific, USA) [23].

\section{ELISA (enzyme-linked immunosorbent assay)}

Levels of IL-2, IL-17, and IFN- $\gamma$ were examined by ELISA assay referring to its instructions. The ELISA kits for IFN- $y$, IL-2, and IL-17 were all purchased from Invitrogen ${ }^{\text {TM }}$ (Thermo Fisher Scientific, USA) [24].

\section{Flow cytometry}

Six days after transplantation, the recipient mice were sacrificed, and spleen and kidney samples were collected and prepared into single-cell suspension. Cells were incubated with anti-CD4-APC antibody and anti-Foxp3-PE antibody following the instructions (eBiosciences). Data were collected and analyzed with flow cytometry (FACSCalibur, BD Biosciences, USA) [25].

\section{Cell transfection}

The LV, LV-DANCR, LV-shRNA, LV-shDANCR were cloned into a lentiviral vector and transferred into 293T cells. After that, the obtained virus particles were used to infect BMMSCs or BMMSC-Ex. 
For the BMMSCs-LV-Ex group, BMMSCs-LV-DANCR-Ex group, BMMSCs-LV-shRNA-Ex group, and BMMSCs-LV-shDANCR-Ex group, the LV, LV-DANCR, LV-shRNA, or LV-shDANCR was transfected into BMMSCs followed by the collection of BMMSC-Ex.

\section{$\mathrm{CD}^{+} \mathrm{T}$ cell isolation}

$\mathrm{CD}^{+}{ }^{+} \mathrm{T}$ cells were isolated from spleens of healthy C57BL/6 mice using Dynabeads ${ }^{\mathrm{TM}}$ Untouched $^{\mathrm{TM}}$ Mouse CD4 Cells Kit (Thermo Fisher Scientific, USA) referring to the instructions. CD4 ${ }^{+} \mathrm{T}$ cells were in the supernatant. Then cultured with different concentrations of BMMSC-Ex $(10,50,100 \mathrm{ng} / \mathrm{mL})$ for 2 days [26].

\section{qRT-PCR}

RNA was isolated from BMMSCs, BMMSC-Ex, CD4 ${ }^{+} \mathrm{T}$ cells, spleen and kidney tissues using Trizol (Beyotime Biotechnology, Shanghai). After reverse transcription, DANCR levels were determined using SYBR qPCR Mix (Takara, Japan) on a Real-time fluorescence quantitative PCR instrument (Applied Biosystems, USA). GAPDH was used as an internal reference and the intergroup multiples were calculated by the $2^{-\triangle \Delta C t}$ method.

DANCR: (F: 5'-CGTCTCTTACGTCTGCGGAA-3'

R: 5'-GGACACGTGGTTGCTACAAG-3');

GAPDH: (F: 5'-TGCACCACCAACTGCTTAG-3'

R: 5'-GGATGCAGGGATGATGTTC-3')

\section{RNA pull-down assay}

RNA pull-down assay was performed according to the instructions of the Magnetic RNA-Protein PullDown Kit (Thermo Fisher, USA). CD4 ${ }^{+}$T cells were lysed using RIP buffer containing protease inhibitor and RNase inhibitor. The biotin-labeled IncRNA DANCR (Bio-DANCR) or control (Bio-NC) was captured with beads and then transfected into $\mathrm{CD}^{+} \mathrm{T}$ cell lysate. Western blot was carried out to examine the protein levels of SIRT1.

\section{RNA immunoprecipitation (RIP) assay}

RIP assay was performed according to the instructions of Magna RIP ${ }^{\text {TM }}$ RNA-Binding Protein Immunoprecipitation Kit (Millipore, Germany). CD4 ${ }^{+}$T cells were lysed using RIP buffer containing protease inhibitor and RNase inhibitor. The $\mathrm{CD} 4^{+} \mathrm{T}$ cell lysate was incubated with magnetic beads coated with anti-SIRT1 (ab32441, 1:30). Normal Rabbit IgG was used as the negative control. After washing and purification, qRT-PCR was carried out to examine DANCR levels. 


\section{Co-immunoprecipitation (Co-IP)}

The FLAG-SMURF2, LV-DANCR (or LV) constructs were transfected into CD $4^{+} \mathrm{T}$ cells and treated with 10 $\mu \mathrm{M}$ MG132 (proteasome inhibitor) for $4 \mathrm{~h}$. Cells were then lysed with RIP Lysis Buffer and incubated with magnetic beads coated with anti-FLAG (SMURF2, Cell Signaling Technology, 1:50). Western blot was carried out to examine the protein levels of SIRT1.

The HA-Ub, FLAG-SMURF2, LV-DANCR (or LV) constructs were transfected into CD $4^{+} \mathrm{T}$ cells and treated with $10 \mu \mathrm{M}$ MG132 for $4 \mathrm{~h}$. Cells were then lysed with RIP Lysis Buffer and incubated with magnetic beads coated with anti-HA-Ub (ab3341, 1:1000). Western blot was carried out to examine the protein levels of SIRT1.

\section{Statistical analysis}

Analyses were performed with GraphPad Prism 8.01. The difference was assessed by Student's t-test or one-way ANOVA followed by the LSD post hoc test. $P<0.05$ was considered statistically significant [27].

\section{Results}

\section{Characteristics of BMMSC-EX.}

To be first, the Characteristics of BMMSC-Ex were observed. Analysis of TEM and NTA showed that BMMSC-Ex was cup-shaped with a diameter of $~ 100 \mathrm{~nm}$ (Figure 1A, 1B). Besides, BMMSC-Ex expressed CD9, TSG101 and CD63, but did not express calnexin (Figure 1C), which up to identification criteria of exosomes.

\section{BMMSC-Ex improved kidney transplant rejection in mice.}

To investigate the effect of BMMSC-Ex on kidney transplant rejection in mice, we established mouse model of isogenic kidney transplantation and allograft kidney transplantation, and BMMSC-Ex was infused through tail vein. It could be seen that the inflammatory response was reduced after injection of BMMSC-Ex compared with the allograft group (Figure $2 \mathrm{~A})$. Also, $\mathrm{CD} 4^{+} \mathrm{T}$ cell infiltration was significantly up-regulated $(P<0.001)$ in the allograft group, and significantly down-regulated $(P<0.01)$ in the allograft + BMMSC-Ex group (Figure 2B). Besides, the $\mathrm{SCr}$ levels were also increased $(P<0.001)$ in the allograft group, while the injection of BMMSC-Ex decreased SCr levels $(P<0.01)$ (Figure $2 \mathrm{C}$ ). ELISA results showed that the expression of rejection-related factors (IFN- $\gamma$, IL-2 and IL-17) in plasma of allograft group was significantly up-regulated $(P<0.001$ or $P<0.01)$, while the expression levels were decreased $(P<0.01)$ after injection of BMMSC-Ex. (Figure 2D-2F). Results indicated that BMMSC-Ex could improve the rejection of kidney transplantation in mice.

\section{BMMSC-Ex increased Treg cells in kidney transplantation mice.}


Studies have indicated that Treg cells could reduce transplant rejection $[4,5]$. We aimed to explore whether BMMSC-Ex improved kidney transplant rejection in mice by increasing Treg cells. Mice were grouped as described in Figure 2. As shown in Figure 3A and Figure 3B, in spleen tissues and transplanted kidney tissues, Treg cells $\left(\mathrm{CD}^{+} \mathrm{FoxP}^{+} \mathrm{T}\right)$ were both increased in the allograft group, and the injection of BMMSC-Ex further increased its proportion. Data demonstrated that BMMSC-Ex might improve kidney transplant rejection in mice by up-regulating the proportion of Treg cells.

\section{Interference with DANCR reversed the promoting effect of BMMSC-Ex on Treg cell differentiation.}

A large number of literatures has shown that BMMSC-Ex can promote the differentiation of CD $4^{+} \mathrm{T}$ cells into Treg cells $[9,10]$. Figure 4A and Figure 4B showed that overexpressing DANCR up-regulated DANCR levels $(P<0.01)$ and interference with DANCR down-regulated DANCR levels $(P<0.01)$ in BMMSCs and BMMSC-Ex. Then, $C D 4^{+} T$ cells were isolated followed by the addition of different concentrations of BMMSC-Ex $(10,50,100 \mathrm{ng} / \mathrm{mL})$ and cultured for 2 days. QRT-PCR results showed that the addition of BMMSC-Ex ( $\geq 50 \mathrm{ng} / \mathrm{mL}$ ) promoted the expression of DANCR (Figure 4C). The LV-DANCR (or LV) was transfected into BMMSCs and then BMMSC-Ex was collected. Figure 4D showed that the addition of BMMSC-Ex increased DANCR levels, and overexpressing DANCR further promoted DANCR expression. The LV-shDANCR (or LV-shRNA) was transfected into BMMSCs and then BMMSC-Ex was collected. Figure 4E showed that the addition of BMMSC-Ex increased DANCR levels, and interference with DANCR reversed the effect of BMMSC-Ex. After inducing Treg cell differentiation, flow cytometry and qRT-PCR results showed that BMMSC-Ex promoted the proportion of Treg cells as well as the levels of Foxp3 and IL-10, while interference with DANCR reversed the effect of BMMSC-Ex (Figure 4F, 4G).

\section{DANCR bound to SIRT1 to inhibit its expression.}

Elimination of SIRT1 has been reported to promote differentiation of $\mathrm{CD} 4^{+} \mathrm{T}$ cells into Treg cells, and inhibition of SIRT1 can improve the survival rate of transplanted kidneys $[17,18]$. Besides, through RPISeq software prediction, we found that there might be an interaction between DANCR and SIRT1. RNA pull-down assay showed that compared with Bio-NC, a large amount of SIRT1 was detected in the BioDANCR pull-down complex (Figure 5A). RIP assay showed that DANCR was significantly enriched in SIRT1 precipitation (Figure 5B). In addition, Interfering with DANCR did not significantly affect the expression of SIRT1 mRNA, but significantly promoted the expression of SIRT1 protein (Figure 5C). Similarly, overexpressing DANCR did not significantly affect the expression of SIRT1 mRNA, but significantly inhibited the expression of SIRT1 protein (Figure 5D). Data demonstrated that DANCR might inhibit SIRT1 expression by binding to SIRT1.

\section{DANCR accelerated SIRT1 degradation and promoted E3 ubiquitin ligase SMURF2-mediated ubiquitylation.}

In the following work, we investigated how DANCR affected SIRT1 protein levels. CD $4^{+} \mathrm{T}$ cells were infected with LV-DANCR or LV, and then treated with $10 \mu \mathrm{g} / \mathrm{mL} \mathrm{CHX}$ (cycloheximide, protein synthesis 
inhibitor) for 0, 2, 4, $8 \mathrm{~h}$. Figure 6A showed that overexpression of DANCR promoted the degradation of SIRT1 protein. Then, cells were treated with (or without) $10 \mu \mathrm{M}$ of MG132 for $4 \mathrm{~h}$. Western blot results indicated that LV-DANCR-mediated degradation of SIRT1 protein was completely inhibited by MG132 (Figure 6B). We speculated whether DANCR accelerated the degradation of SIRT1 by affecting its ubiquitination. Co-IP results showed that in LV-DANCR transfected cells, the levels of SIRT1 pulled down by Flag-SMURF2 were increased (Figure 6C). Next, immunoprecipitation (IP) was performed with anti-HA (Ub) Antibody, followed by western blot (IB) with anti-SIRT1 Antibody. The results showed that the addition of Flag-SMURF2 promoted SIRT1 ubiquitination, and the overexpression of DANCR further upregulated the level of Flag-SMURF2-mediated SIRT1 ubiquitination (Figure 6D). Results demonstrated that overexpression of DANCR might accelerate SIRT1 protein degradation by promoting SMURF2mediated SIRT1 ubiquitination.

\section{After interference with DANCR, the collected BMMSC-Ex inhibited Treg cell differentiation by up-regulating SIRT1 expression in CD4+ $T$ cells.}

Next, we explored whether BMMSC-Ex (after interference with DANCR) affected Treg differentiation by regulating SIRT1 protein. Figure 7A showed that in CD $4^{+} \mathrm{T}$ cells, LV-shDANCR-Ex promoted the expression of SIRT1, and co-culture with LV-shSIRT1 reversed the effect. Flow cytometry results showed that Treg cells were inhibited by LV-shDANCR-Ex and the effect was reversed by co-culture with LV-shSIRT1 (Figure 7B). QRT-PCR results showed that LV-shDANCR-Ex inhibited the expression of Foxp3 and IL-10 in CD4+ cells, and co-culture with LV-shSIRT1 increased their levels (Figure 7C, 7D).

\section{After interference with DANCR, the collected BMMSC-Ex aggravated kidney transplant rejection in mice.}

To investigate the effect of BMMSC-Ex interfering with DANCR on kidney transplant rejection in mice in vivo, exosomes collected after transfection of BMMSCs with or without LV-shRNA or LV-shDANCR were injected into mice via tail vein. In spleen and transplanted kidney tissues, injection of BMMSC-Ex promoted the expression of DANCR, and LV-shDANCR-Ex reversed the effect (Figure 8A). Western blot results showed that injection of BMMSC-Ex inhibited the expression of SIRT1 in the spleen and kidney, while LV-shDANCR-Ex reversed the effect (Figure 8B). HE staining of kidney tissues showed that the inflammatory response was reduced after injection of BMMSC-Ex, and this effect was reversed by injection of BMMSCs-LV-shDANCR-Ex (Figure 8C). CD4 immunohistochemistry of kidney tissues showed that the injection of BMMSC-Ex significantly reduced $C D 4^{+} T$ cell infiltration, which was reversed by LVshDANCR-Ex (Figure 8D). Besides, the injection of BMMSC-Ex reduced the expression of serum creatinine (SCr) as well as the expression of rejection-related factors (IFN-y, IL-2, and IL-17) in plasma, and the effect was reversed by injection of LV-shDANCR-Ex (Figure 8E-8H). Figure $8 \mathrm{I}$ results showed that the injection of BMMSC-Ex increased the proportion of Treg cells in the spleen and transplanted kidney tissues, while the injection of LV-shDANCR-Ex reversed this effect.

\section{Discussion}


The transplanted kidney will be "attacked" by immune-active cells, mainly lymphocytes, which is called kidney rejection. Immunosuppressant is often used in clinical practice to prevent the immune rejection of kidney transplants, but it will produce immune-mediated injury and adverse reactions [28]. Therefore, it is very necessary to explore the mechanism of immune tolerance in kidney transplantation. In the present study, the mouse kidney transplantation model was established and we found that BMMSC-Ex could improve kidney transplant rejection and up-regulate the proportion of Treg cells in kidney transplantation mice. At the same time, we also found that DANCR could bind to SIRT1, promote its ubiquitination and accelerate its degradation. In addition, after interference with DANCR, BMMSC-Ex inhibited the differentiation of $\mathrm{CD} 4^{+} \mathrm{T}$ cells into Treg cells by up-regulating the expression of SIRT1 and finally aggravated kidney transplant rejection in mice.

Treg cells play an important role in the resistance to immune rejection in kidney transplantation. Treg cells can specifically inhibit autoreactive $T$ cells and are important cells involved in peripheral immune tolerance [29]. It has been reported that injection of MSCs can improve kidney transplant rejection and induce immune tolerance by increasing the proportion of Treg cells $[5,30]$. The role of exosomes in organ transplantation has been demonstrated in a large number of literatures. For example, it has been reported that the addition of Treg cell-derived exosomes promotes immune tolerance in kidney transplantation [7, 8]. Other studies show that BMMSCs-derived exosomes promote the differentiation of $\mathrm{CD}^{+}{ }^{+} \mathrm{T}$ cells into Treg cells $[19,26]$. In our study, we found that injection of BMMSC-Ex could reduce the inflammatory response, the infiltration of $\mathrm{CD} 4^{+} \mathrm{T}$ cells, decrease the expression levels of serum $\mathrm{SCr}$ and plasma rejection related factors (IFN-y, IL-2, and IL-17), and improve the rejection of kidney transplantation in mice. Meanwhile, injection of BMMSC-Ex also up-regulated the proportion of Treg cells in kidney transplantation mice. The results indicated that BMMSCs-derived exosomes might promote kidney transplantation immune tolerance by promoting $\mathrm{CD} 4^{+} \mathrm{T}$ cell differentiation into Treg cells.

Exosomes mediate intercellular communication by delivering IncRNA, mRNA, and other signaling molecules [11]. LncRNA has been reported to regulate the immune system [31]. Studies have shown that IncRNA DANCR can protect kidney against injury. It can inhibit renal cell carcinoma, promote the viability of LPS-induced renal tubular epithelial cells and inhibit cell apoptosis $[12,13]$. Furthermore, IncRNA DANCR can be expressed in BMMSCs and can be encapsulated in exosomes $[14,15]$. In our study, after interfering with DANCR, the expression of DANCR was decreased, while it was increased after overexpression of DANCR in BMMSCs and BMMSC-Ex. Besides, the addition of BMMSC-Ex further promoted the expression of DANCR after overexpressing DNACR, while interference with DANCR downregulated its expression. In addition, after interference with DANCR, Treg cells was decreased, as well as the expression of Foxp3 and IL-10. Data suggested that interference with DANCR might inhibit the promotion of BMMSC-Ex on Treg cell differentiation.

Through RPISeq software, DANCR was predicted to interact with SIRT1. Sirtuin-1 (SIRT1), a member of the Sirtuin family, is an important target for immunotherapy due to its deacetylation of transcription factor Foxp3 and thymoretinic acid receptor-associated orphan receptor y (RORYt). It has been reported that inhibition of SIRT1 can improve the survival rate and function of transplanted kidneys [18, 32]. Also, 
an inhibitor of sirtuin-1 (Ex-527) significantly increases the proportion of Treg cells, as well as Foxp3 expression levels [23]. RNA pull-down, RIP, Co-IP, and other experiments confirmed the binding relationship between DANCR and SIRT1, and DANCR accelerated the degradation of SIRT1 by promoting its ubiquitination. Furthermore, after interference with DANCR, BMMSC-Ex inhibited the differentiation of $\mathrm{CD} 4^{+} \mathrm{T}$ cells into Treg cells by up-regulating the expression of SIRT1 and aggravated the rejection of kidney transplantation in mice.

Overall, our experiments firstly confirmed that IncRNA DANCR in BMMSC-Ex could promote immune tolerance of kidney transplantation partly by down-regulating SIRT1 expression, and promoting $\mathrm{CD} 4^{+} \mathrm{T}$ cells to differentiate into Treg cells. We also found that DANCR could bind to SIRT1, promote E3 ubiquitin ligase SMURF2-mediated ubiquitination and accelerate the degradation of SIRT1.

\section{Declarations}

\section{Ethics approval and consent to participate}

Our study was approved by Zhengzhou University People's Hospital.

The experimental procedures were approved by the Medical Ethics review committee of Zhengzhou University People's Hospital

\section{Consent for publication}

The study was undertaken with the patient's consent.

\section{Availability of data and materials}

Not applicable.

\section{Conflict of Interest}

The authors declare that they have no conflict of interest.

\section{Competing Interests}

The authors declare that they have no competing interests.

\section{Funding}

This study was supported by the foundation of Medical Science and Technology of Henan Province, No. LHGJ20200046.

\section{Authors' contributions}


XW\&TY put forward the concept of the study, designed the study, prepared the manuscript and contributed to the statistical analysis. ZW\&JW contributed to the data acquisition. XT\&GC contributed to the quality control of data and algorithms. YG analyzed the data and interpretation.

FS edited the manuscript. TY put forward the concept of the study, contributed to the data analysis and interpretation and reviewed the manuscript. All authors read and approved the final manuscript

\section{Acknowledgements}

Not applicable.

\section{References}

1. Mehta R., Bhusal S., Randhawa P. ,et al. (2018).Short-term adverse effects of early subclinical allograft inflammation in kidney transplant recipients with a rapid steroid withdrawal protocol. Am J Transplant. 7:1710-1717.

2. Xu Q.X., Qiu X.Y., Jiao Z. ,et al. (2018).FOXP3 rs3761549 polymorphism predicts long-term renal allograft function in patients receiving cyclosporine-based immunosuppressive regimen. Gene.93-100.

3. Georgiev P., Charbonnier L.M., Chatila T.A. (2019).Regulatory T Cells: the Many Faces of Foxp3. J Clin Immunol. 7:623-640.

4. Liao T., Xue Y., Zhao D. ,et al. (2017).In Vivo Attenuation of Antibody-Mediated Acute Renal Allograft Rejection by Ex Vivo TGF- $\beta$-Induced CD4(+)Foxp3(+) Regulatory T Cells. Front Immunol.1334.

5. He Y., Zhou S., Liu H. ,et al. (2015).Indoleamine 2, 3-Dioxgenase Transfected Mesenchymal Stem Cells Induce Kidney Allograft Tolerance by Increasing the Production and Function of Regulatory $\mathrm{T}$ Cells. Transplantation. 9:1829-1838.

6. Pang X.L., Wang Z.G., Liu L. ,et al. (2019).Immature dendritic cells derived exosomes promotes immune tolerance by regulating T cell differentiation in renal transplantation. Aging (Albany NY). 20:8911-8924.

7. Yu X., Huang C., Song B. ,et al. (2013).CD4+CD25+ regulatory T cells-derived exosomes prolonged kidney allograft survival in a rat model. Cell Immunol. 1-2:62-68.

8. Aiello S., Rocchetta F., Longaretti L. ,et al. (2017).Extracellular vesicles derived from T regulatory cells suppress T cell proliferation and prolong allograft survival. Sci Rep. 1:11518.

9. Ji L., Bao L., Gu Z. ,et al. (2019).Comparison of immunomodulatory properties of exosomes derived from bone marrow mesenchymal stem cells and dental pulp stem cells. Immunol Res. 4-5:432-442. 
10. Li Y., Wang F., Guo R. et al. (2019).Exosomal sphingosine 1-phosphate secreted by mesenchymal stem cells regulated Treg/Th17 balance in aplastic anemia. IUBMB Life. 9:1284-1292.

11. Li P., Kaslan M., Lee S.H. ,et al. (2017).Progress in Exosome Isolation Techniques. Theranostics. 3:789-804.

12. Jin L., Fu H., Quan J. ,et al. (2017). Overexpression of long non-coding RNA differentiation antagonizing non-protein coding RNA inhibits the proliferation, migration and invasion and promotes apoptosis of renal cell carcinoma. Mol Med Rep. 4:4463-4468.

13. Zhao H., Chen B., Li Z. ,et al. (2020).Long Noncoding RNA DANCR Suppressed LipopolysaccharideInduced Septic Acute Kidney Injury by Regulating miR-214 in HK-2 Cells. Med Sci Monit.e921822.

14. Zhang J., Tao Z., Wang Y. (2018).Long non-coding RNA DANCR regulates the proliferation and osteogenic differentiation of human bone-derived marrow mesenchymal stem cells via the p38 MAPK pathway. Int J Mol Med. 1:213-219.

15. Karlsson O., Rodosthenous R.S., Jara C. ,et al. (2016).Detection of long non-coding RNAs in human breastmilk extracellular vesicles: Implications for early child development. Epigenetics. 10:721-729.

16. Chadha S., Wang L., Hancock W.W. ,et al. (2019).Sirtuin-1 in immunotherapy: A Janus-headed target. J Leukoc Biol. 2:337-343.

17. Heyn J., Luchting B., Hinske L.C. ,et al. (2016).miR-124a and miR-155 enhance differentiation of regulatory $T$ cells in patients with neuropathic pain. $J$ Neuroinflammation. 1:248.

18. Levine M.H., Wang Z., Xiao H. ,et al. (2016).Targeting Sirtuin-1 prolongs murine renal allograft survival and function. Kidney Int. 5:1016-1026.

19. Yang R., Huang H., Cui S. ,et al. (2020).IFN-y promoted exosomes from mesenchymal stem cells to attenuate colitis via miR-125a and miR-125b. Cell Death Dis. 7:603.

20. Kou X., Xu X., Chen C. ,et al. (2018).The Fas/Fap-1/Cav-1 complex regulates IL-1RA secretion in mesenchymal stem cells to accelerate wound healing. Sci Trans/ Med. 432.

21. Madeline G. Roman, Lisa C. Flores, Geneva M. Cunningham ,et al. (2020). Thioredoxin overexpression in mitochondria showed minimum effects on aging and age-related diseases in male C57BL/6 mice. APT.20-31.

22. Wu K., Liou H., Lee C. ,et al. (2019).Down-regulation of natural resistance-associated macrophage protein-1 (Nramp1) is associated with 1-methyl-4-phenyl-1,2,3,6-tetrahydropyridine (MPTP)/1-methyl-4phenylpyridinium (MPP)-induced a-synuclein accumulation and neurotoxicity. Neuropathology applied neurobiology. 2:157-173. 
23. Wen-qing Ge, Pan hao, Yu-hua Huang ,et al. (2018).miR-539 Inhibits Inflammation in Renal Transplant Iscemia-Reperfusion Injury via Blocking the MyD88/NF-kB Pathway. Clin Surg Res Commun. 2:14-21.

24. Mitsugu Hachisu, Masahiro Hashizume, Hisashi Kawai ,et al. (2020). Finding prodromal frailty in a community-dwelling healthy older cohort by survey of BDNF or hand grip strength classified by BMI. APT. 3:155-161.

25. Azuma H., Isaka Y., Li X. ,et al. (2008).Superagonistic CD28 antibody induces donor-specific tolerance in rat renal allografts. Am J Transplant. 10:2004-2014.

26. Cosenza S., Toupet K., Maumus M. ,et al. (2018).Mesenchymal stem cells-derived exosomes are more immunosuppressive than microparticles in inflammatory arthritis. Theranostics. 5:1399-1410.

27. Xin Xu, Ting Wei, Weijie Zhong ,et al. (2020).IL-17 regulates the expression of major histocompatibility complex II and VEGF in DLBCL mice on tumor growth. Aging Pathobiology and Therapeutics. 2:96-100.

28. Cornell L.D., Smith R.N., Colvin R.B. (2008).Kidney transplantation: mechanisms of rejection and acceptance. Annu Rev Pathol.189-220.

29. Copsel S., Wolf D., Komanduri K.V. ,et al. (2019).The promise of CD4(+)FoxP3(+) regulatory T-cell manipulation in vivo: applications for allogeneic hematopoietic stem cell transplantation.

Haematologica. 7:1309-1321.

30. Ge W., Jiang J., Arp J. ,et al. (2010). Regulatory T-cell generation and kidney allograft tolerance induced by mesenchymal stem cells associated with indoleamine 2,3-dioxygenase expression.

Transplantation. 12:1312-1320.

31. Atianand M.K., Caffrey D.R., Fitzgerald K.A. (2017).Immunobiology of Long Noncoding RNAs. Annu Rev Immunol.177-198.

32. Yang X., Lun Y., Jiang H. ,et al. (2018).SIRT1-Regulated Abnormal Acetylation of FOXP3 Induces Regulatory T-Cell Function Defect in Hashimoto's Thyroiditis. Thyroid. 2:246-256.

\section{Figures}


A

B

BMMSCs-Ex

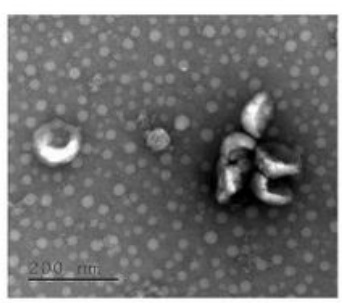

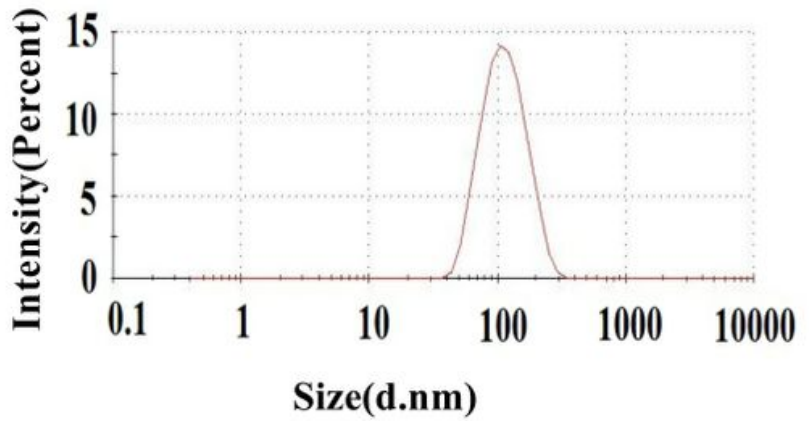

C

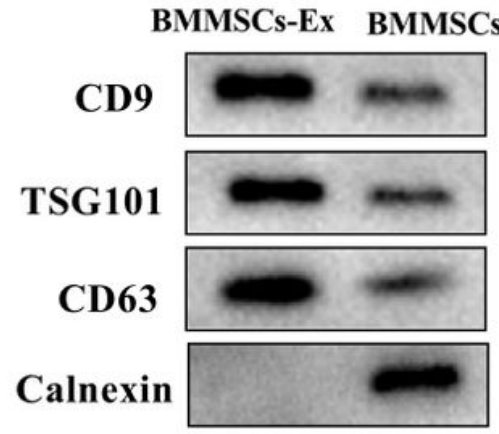

\section{Figure 1}

Characteristics of BMMSC-Ex. BMMSCs were isolated from the femur and tibia of healthy C57BL/ 6 mice and then BMMSC-Ex was collected. (A) The morphology of BMMSC-Ex was observed by transmission electron microscopy (TEM). Scale bar: $200 \mathrm{~nm}$. (B) The size of BMMSC-Ex was determined by nanoparticle tracking analysis (NTA). (C) Exosome markers CD9, TSG101, CD63, and calnexin were detected by Western blot.

A
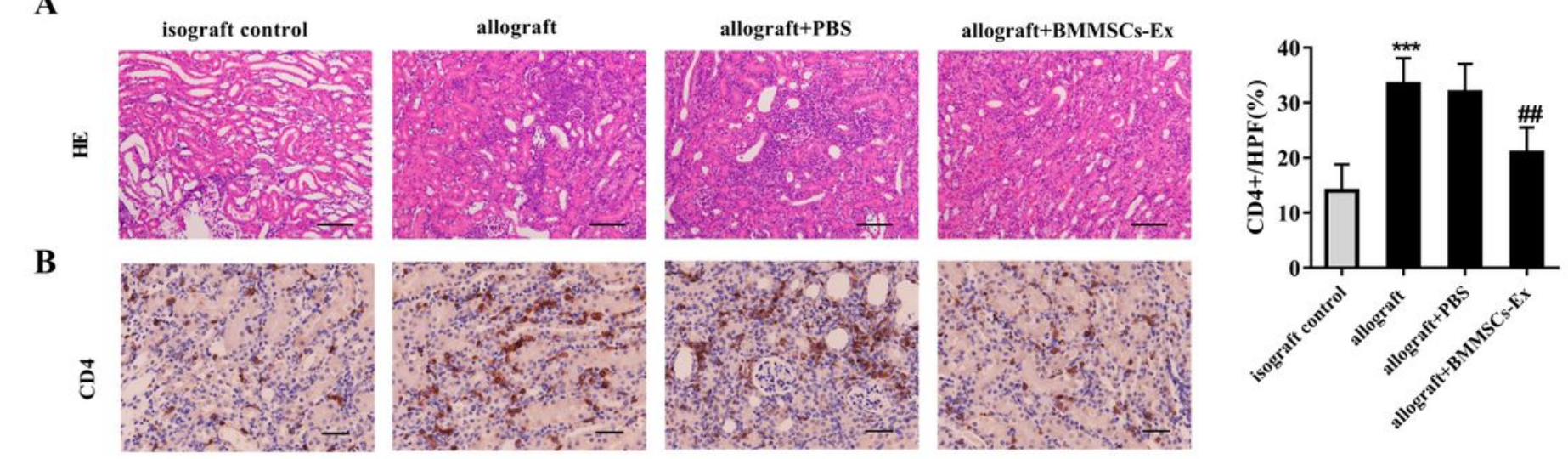

C

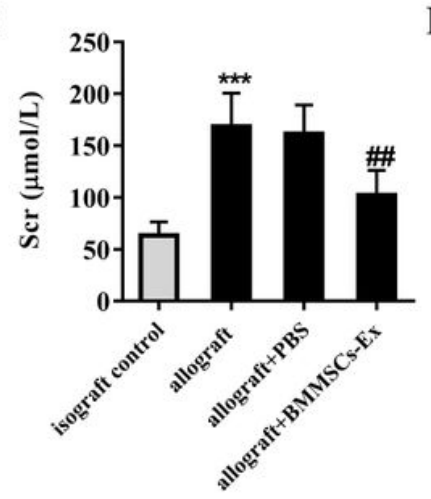

D

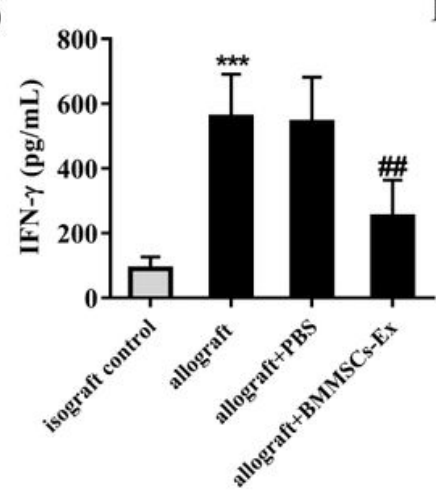

$\mathbf{E}$

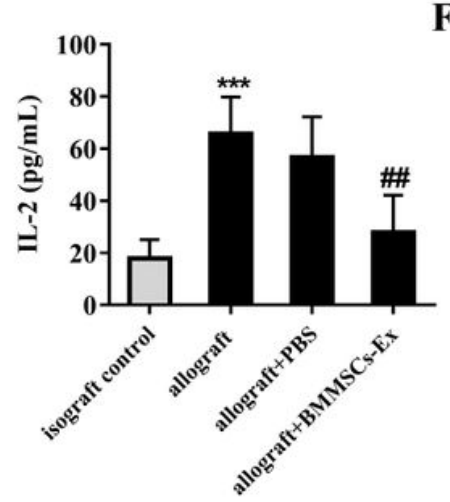

$\mathbf{F}$

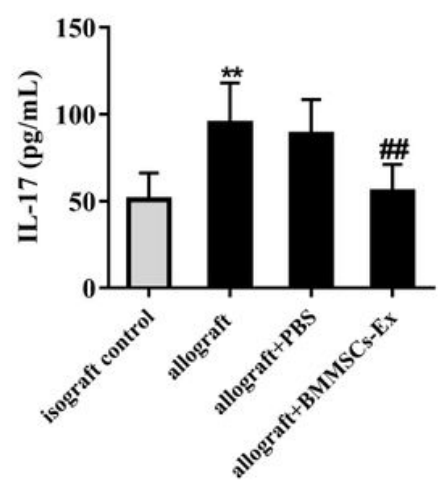

Figure 2

BMMSC-Ex improved kidney transplant rejection in mice. The mouse model of kidney transplantation was established. Mice were divided into four groups ( $n=7$ per group): the isograft control group (isogenic 
kidney transplantation), the allograft group (allograft kidney transplantation), the allograft + PBS group (mice were infused with PBS through caudal vein 1 day before and 1 day after kidney transplantation), the allograft + BMMSC-Ex group (mice were infused with BMMSC-Ex through caudal vein 1 day before and 1 day after kidney transplantation). Six days after transplantation, the recipient mice were sacrificed, the transplanted kidney tissues, serum, and plasma samples were collected for analysis. (A) The inflammation of kidney tissues was observed by HE staining. Scale bar: $100 \mu \mathrm{m}$. (B) The infiltration of CD4+ T cells in kidney tissues was observed by CD4 immunohistochemistry. Scale bar: $40 \mu \mathrm{m}$. (C) Serum creatinine ( $\mathrm{SCr}$ ) levels were measured by an automatic biochemical analyzer. (D-F) The expression of

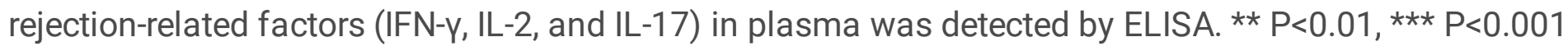
versus the isograft control group; \#\# $\mathrm{P}<0.01$ versus the allograft + PBS group.

A

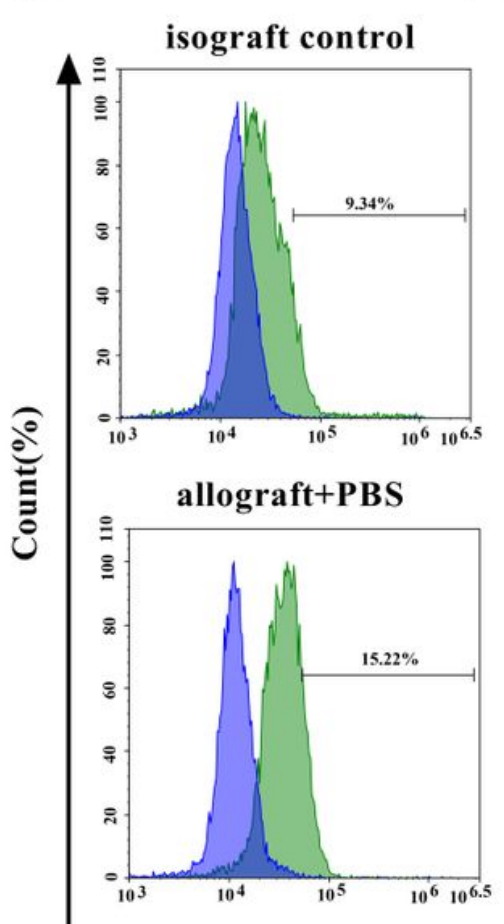

B
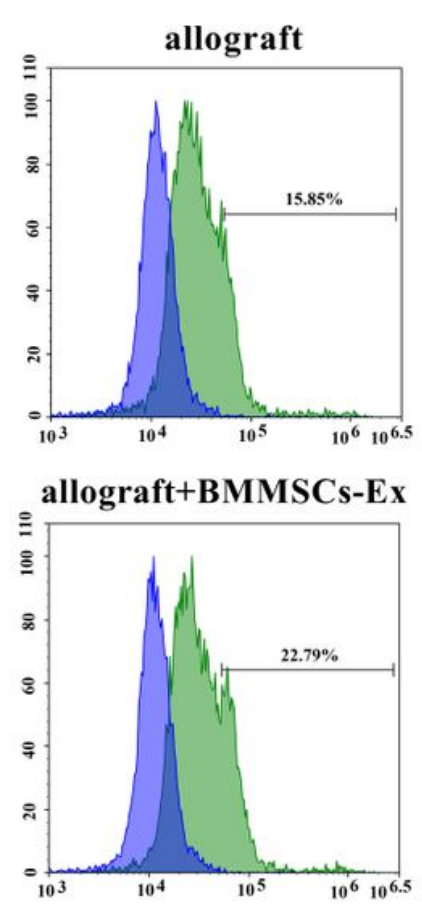

Kidney
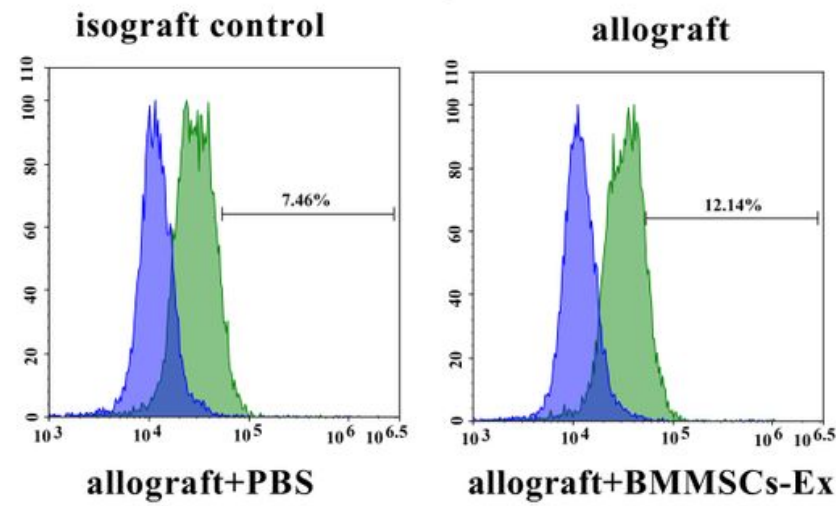

allograft+BMMSCs-Ex
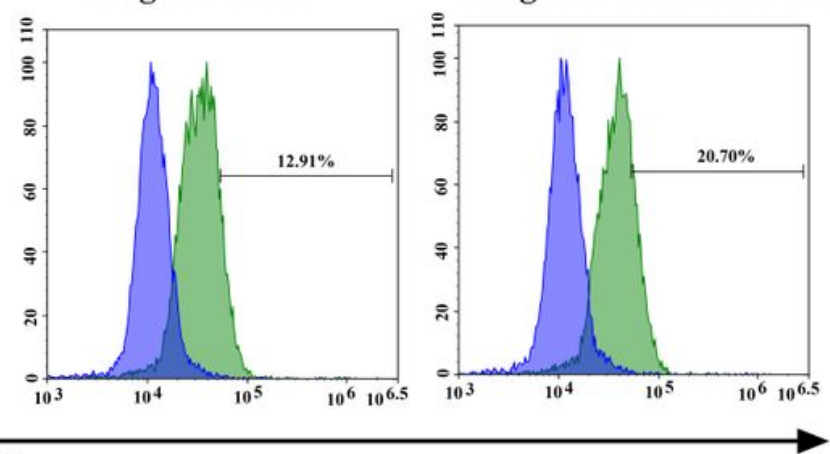

FOXP3
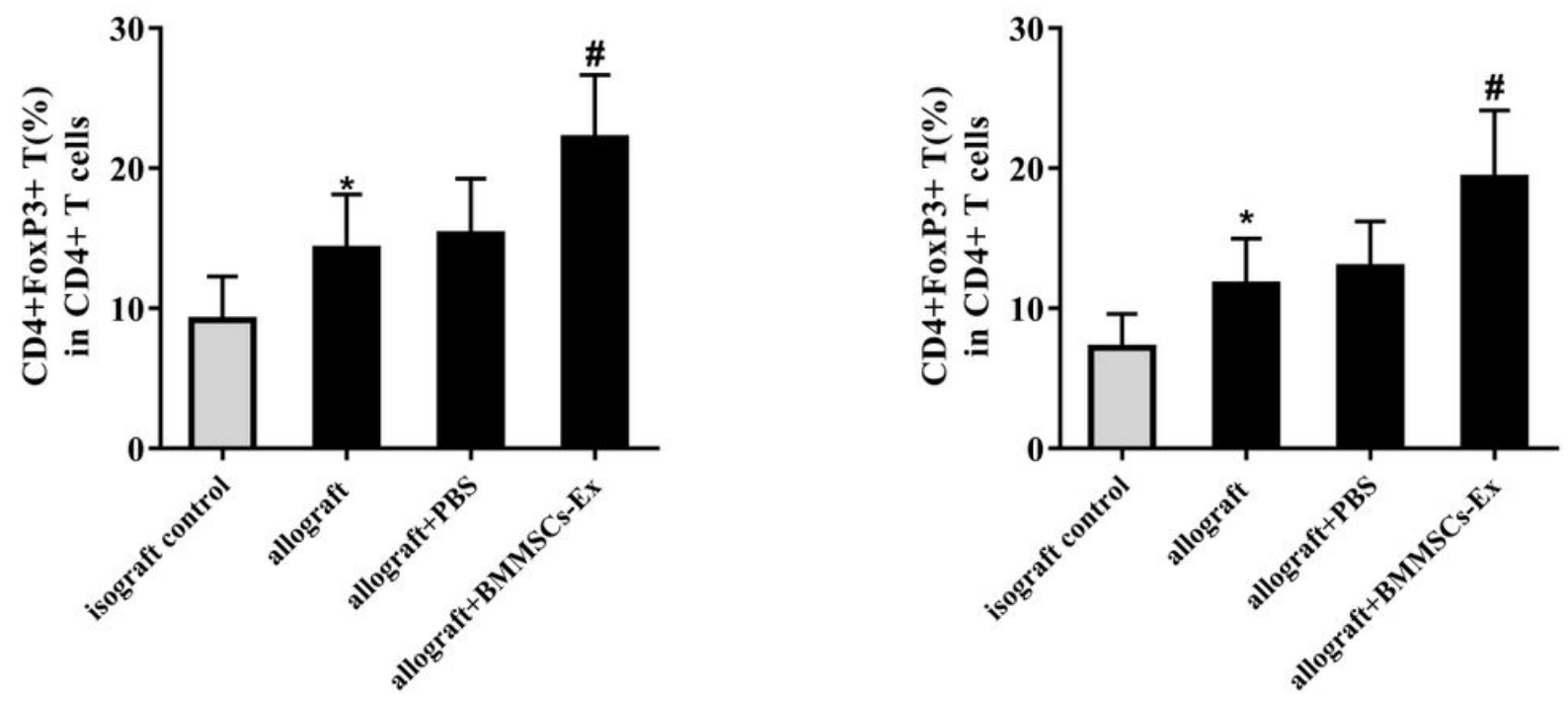
Figure 3

BMMSC-Ex increased the proportion of Treg cells in kidney transplantation mice. Mice were grouped as described in Figure 2. Six days after transplantation, the recipient mice were sacrificed, and spleen and kidney tissues were collected and prepared into single-cell suspension for analysis. (A) The proportion of Treg cells (CD4+ Foxp3+T) in the spleen tissues was determined by flow cytometry. (B) The proportion of Treg cells in transplanted kidney tissues was determined by flow cytometry. ${ }^{*} P<0.05$ versus the isograft control group; \# $\mathrm{P}<0.05$ versus the allograft + PBS group.

A

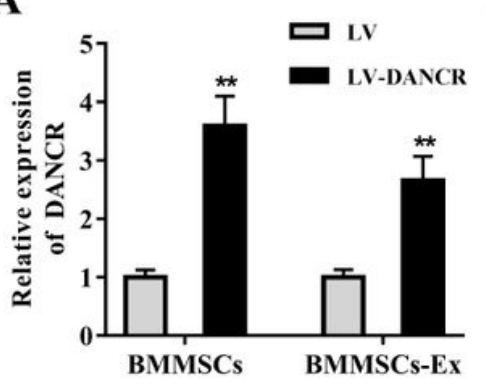

B

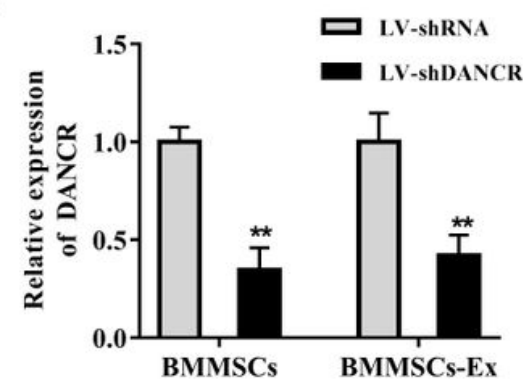

C

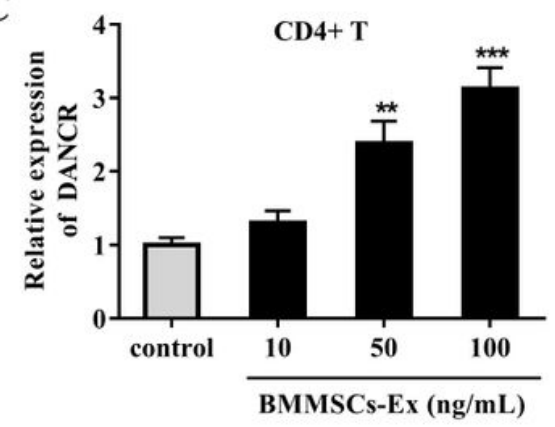

D

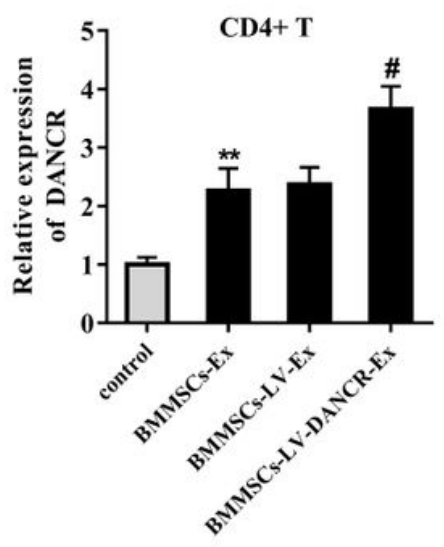

$\mathbf{E}$

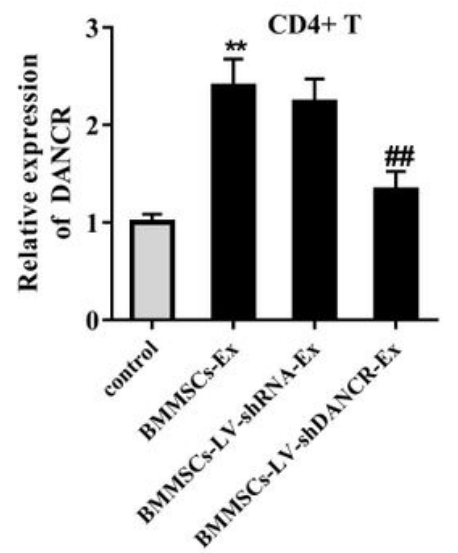

G

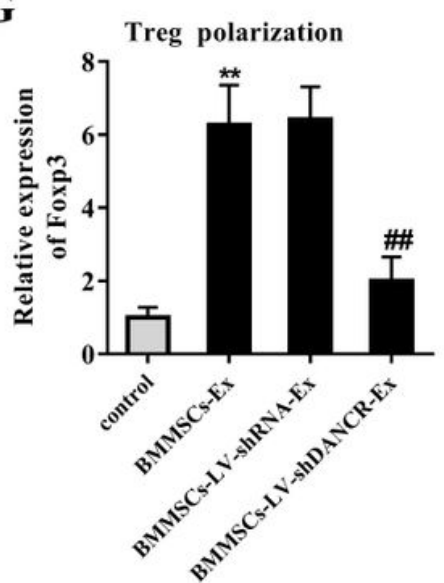

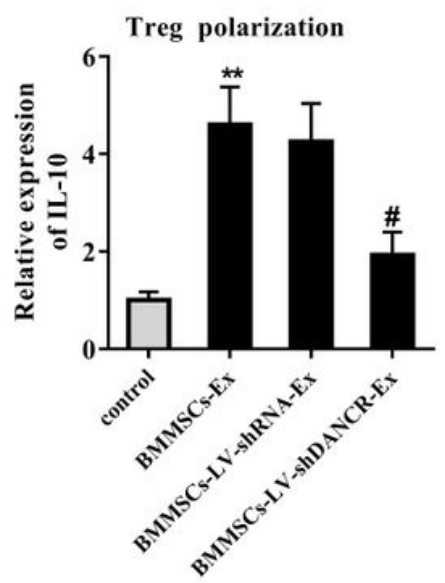

F Treg polarization
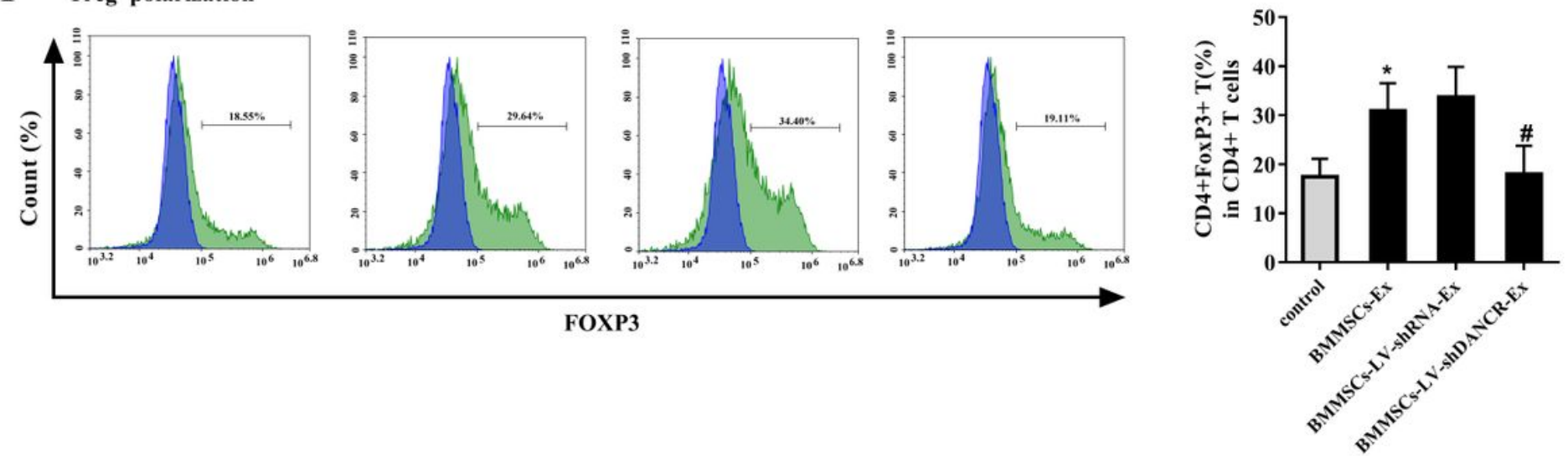

Figure 4

Interference with DANCR reversed the promoting effect of BMMSC-Ex on Treg cell differentiation. BMMSCs were isolated from the femur and tibia of healthy C57BL/ 6 mice and transfected with LV- 
DANCR or LV, followed by the collection of BMMSC-Ex. (A) The expression of DANCR in BMMSCs and BMMSC-Ex was detected by qRT-PCR. ${ }^{* *} \mathrm{P}<0.01$ versus the LV group. BMMSCs were transfected with LVshDANCR or LV-shRNA, followed by the collection of BMMSC-Ex. (B) The expression of DANCR in BMMSCs and BMMSC-Ex was detected by qRT-PCR. ${ }^{* *} P<0.01$ versus the LV-shRNA group. CD 4+ T cells were isolated from spleens of healthy $\mathrm{C} 57 \mathrm{BL} / 6$ mice using a mouse $\mathrm{CD} 4+\mathrm{T}$ cell isolation kit, and then the collected BMMSC-Ex $(10,50,100 \mathrm{ng} / \mathrm{mL})$ was added for co-culture for 2 days. (C) The expression of DANCR in CD4+ T cells was detected by qRT-PCR. ${ }^{*} P<0.01, * \star * ~ P<0.001$ versus the control group. CD 4+ T cells were grouped: Control (CD4+ T cells), BMMSC-Ex (50 ng/mL BMMSC-Ex was added to CD4+ T cells), BMMSCS-LV-Ex (BMMSCs were transfected with LV, and BMMSC-Ex was collected and added to CD4+ T cells), BMMSCs-LV-DANCR-Ex (BMMSCs were transfected with LV-DANCR, and BMMSC-Ex was collected and added to CD4+ T cells). (D) The expression of DANCR in CD4+ T cells was detected by qRTPCR. ${ }^{*} \mathrm{P}<0.01$ versus the control group; \# $\mathrm{P}<0.05$ versus the BMMSCs-LV-Ex group. CD4+ $T$ cells were grouped: Control (CD4+ T cells), BMMSC-Ex (50 ng/mL BMMSC-Ex was added to CD4+ T cells), BMMSCs-LV-shRNA-Ex (BMMSCs were transfected with LV-shRNA, and BMMSC-Ex was collected and added to CD4+ T cells), BMMSCs-LV-shDANCR-Ex (BMMSCs were transfected with LV-shDANCR, and BMMSC-Ex was collected and added to CD4+ T cells). (E) The expression of DANCR in CD4+ T cells was detected by qRT-PCR. ** $P<0.01$ versus the control group; \#\# $P<0.01$ versus the BMMSCs-LV-shRNA-Ex group. CD4+ T cells were grouped: control, BMMSC-Ex, BMMSCS-LV-shRNA-Ex, BMMSCs-LV-shDANCREx. Cells were all cultured in the medium supplemented with Treg cell differentiation inducer $(5 \mu \mathrm{g} / \mathrm{mL}$ anti-CD3, $5 \mu \mathrm{g} / \mathrm{mL}$ anti-CD28, $2 \mathrm{ng} / \mathrm{mL}$ TGF $\beta 1$, and $10 \mathrm{U} / \mathrm{mL}$ IL-2) for 4 days. (F) The proportion of Treg cells in CD4+ T cells was determined by flow cytometry. ${ }^{*} P<0.05$ versus the control group; $\# P<0.05$ versus the BMMSCs-LV-shRNA-Ex group. (G) The expression of Foxp3 and IL-10 in CD4+ T cells was detected by qRT-PCR. ** $P<0.01$ versus the control group; \# $P<0.05$, \#\# $P<0.01$ versus the BMMSCs-LVshRNA-Ex group. 
A

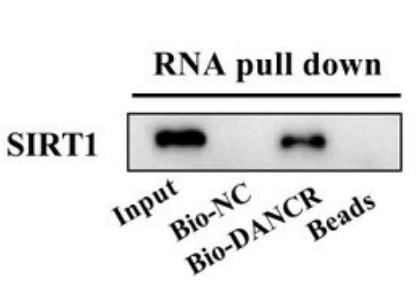

B

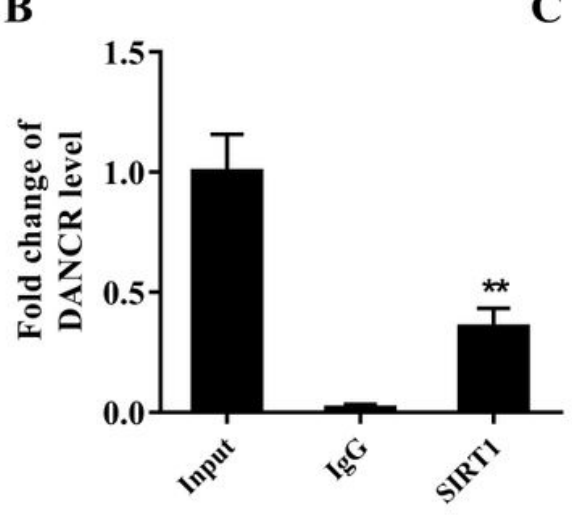

D
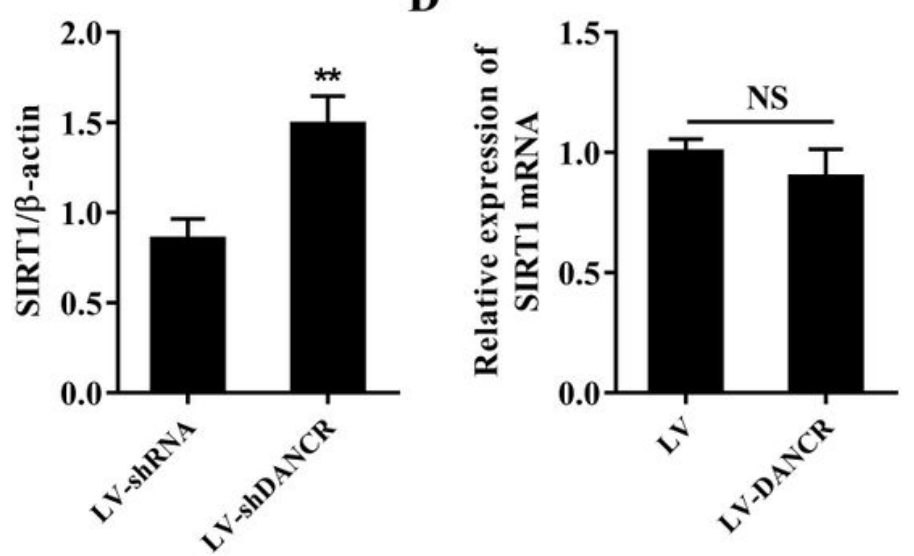

C

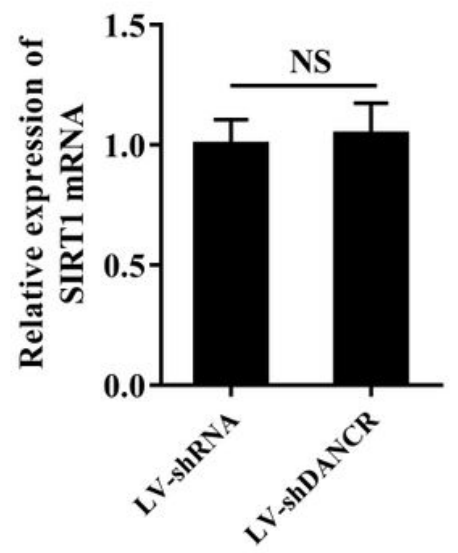

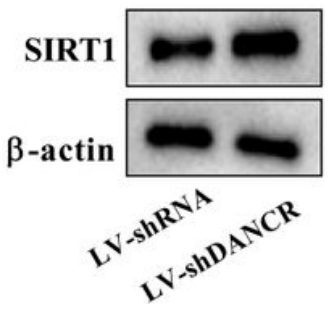
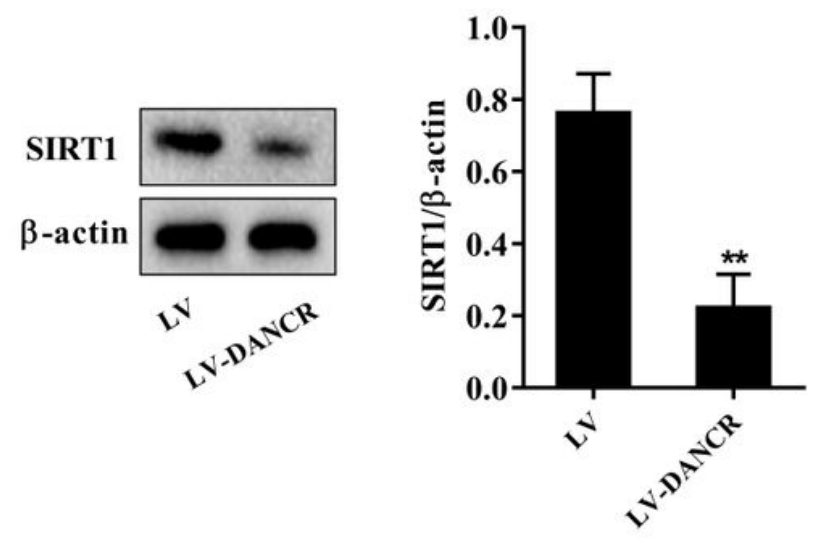

Figure 5

DANCR bound to SIRT1 to inhibit its expression. (A-B) RNA pull-down and RIP were conducted to confirm the binding relationship between DANCR and SIRT1. ** $P<0.01$ versus the IgG group. CD 4+ $T$ cells were transfected with LV-shRNA or LV-shDANCR. (C) SIRT1 levels were detected by qRT-PCR and Western blot. NS $P>0.05, * * P<0.01$ versus the LV-shRNA group. CD4+ T cells were transfected with LV or LV-DANCR. (D) SIRT 1 levels were detected by qRT-PCR and Western blot. NS $P>0.05, * * P<0.01$ versus the LV group. 
A

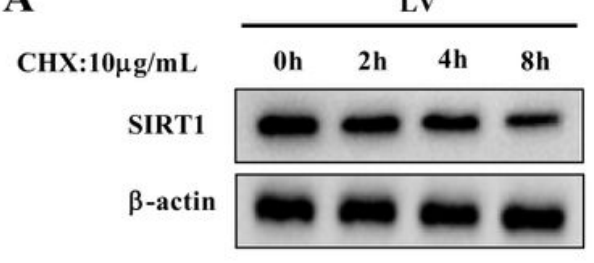

B
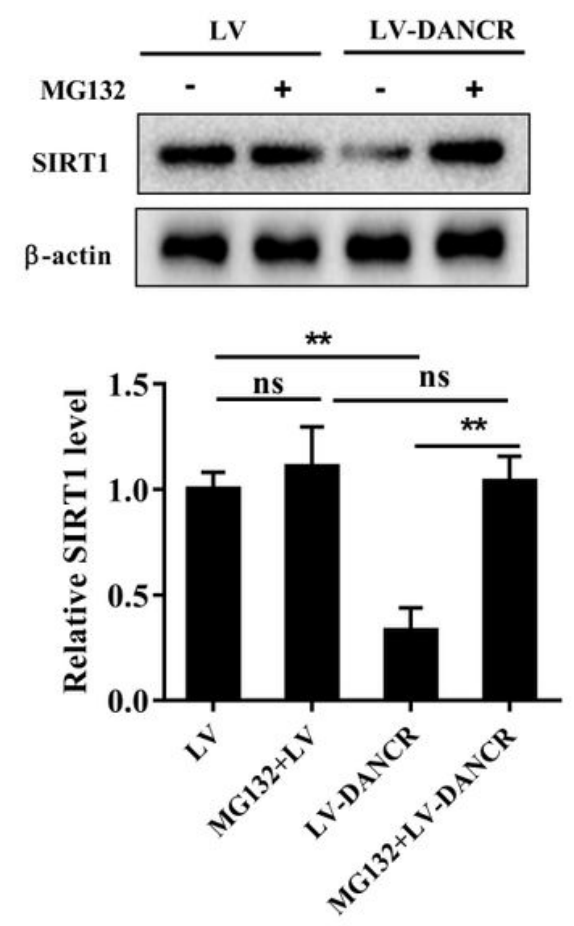

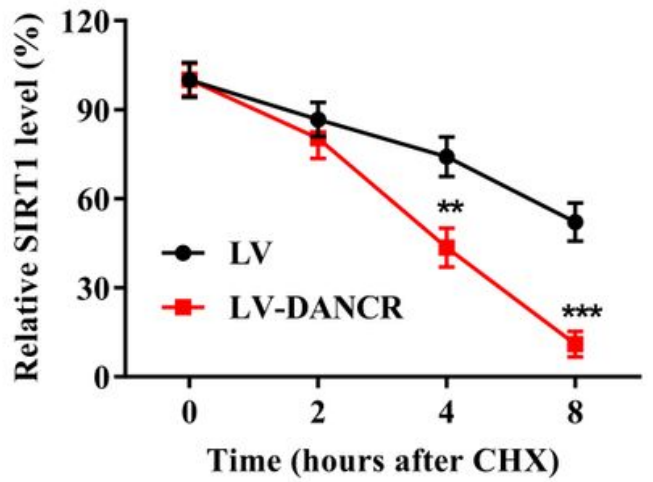

C

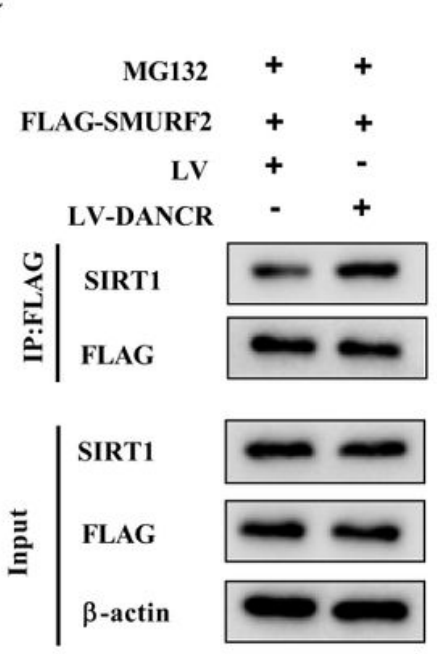

D
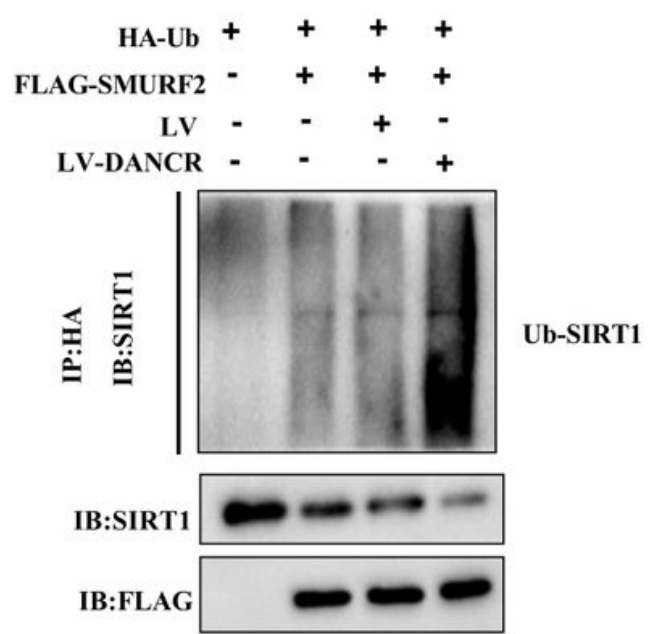

\section{Figure 6}

DANCR accelerated SIRT1 degradation and promoted E3 ubiquitin ligase SMURF2-mediated ubiquitination. CD4+ T cells were infected with LV-DANCR or LV and then treated with $10 \mu \mathrm{g} / \mathrm{mL} \mathrm{CHX} \mathrm{for}$ $0,2,4,8 \mathrm{~h}$. (A) The protein level of SIRT1 was detected by Western blot. ${ }^{*} \mathrm{P}<0.01,{ }^{\star *} \mathrm{P}<0.001$ versus the LV group. CD4+ T cells were infected with LV-DANCR or LV and then treated with (or without) $10 \mu \mathrm{M}$ MG132 for $4 \mathrm{~h}$. (B) The protein level of SIRT1 was determined by Western blot. ${ }^{*} P<0.01$ versus the LV group or LV-DANCR group; ns $P>0.05$ versus the LV group or MG132 + LV group; $* \star P<0.01$ versus the LV group or the LV-DANCR group. CD4+ T cells were infected with FLAG-SMURF2, LV-DANCR or LV, and then treated with $10 \mu \mathrm{M} \mathrm{MG132}$ for $4 \mathrm{~h}$. (C) Co-IP assay was conducted to detect the interaction between SIRT1 and E3 ubiquitin ligase SMURF2. CD4+ T cells were infected with HA-Ub, FLAG-SMURF2, LVDANCR, or LV, and then treated with $10 \mu \mathrm{M} M G 132$ for $4 \mathrm{~h}$. (D) Cell lysates were immunoprecipitated (IP) with anti-HA (Ub) Antibody and then immunoblotted (IB) with anti-SIRT1 Antibody. 
A

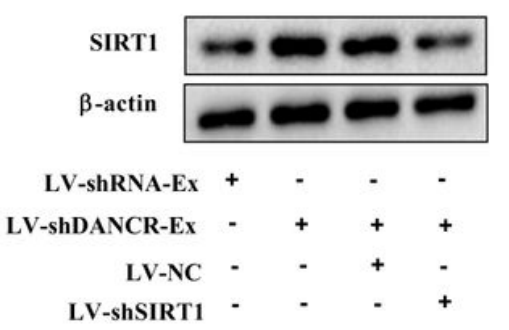

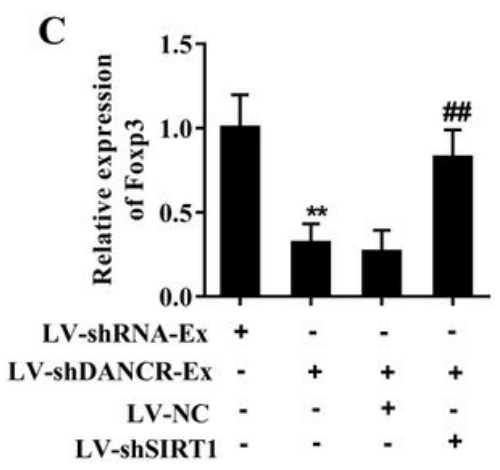

D

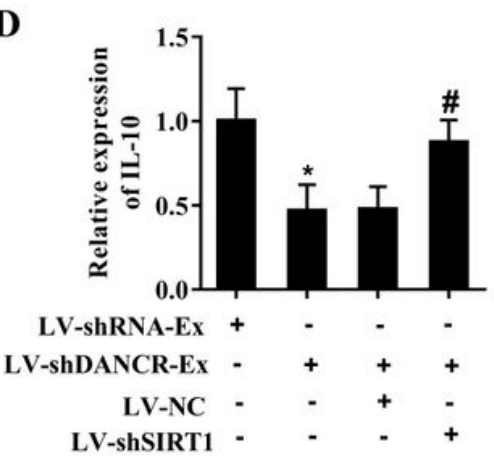

B
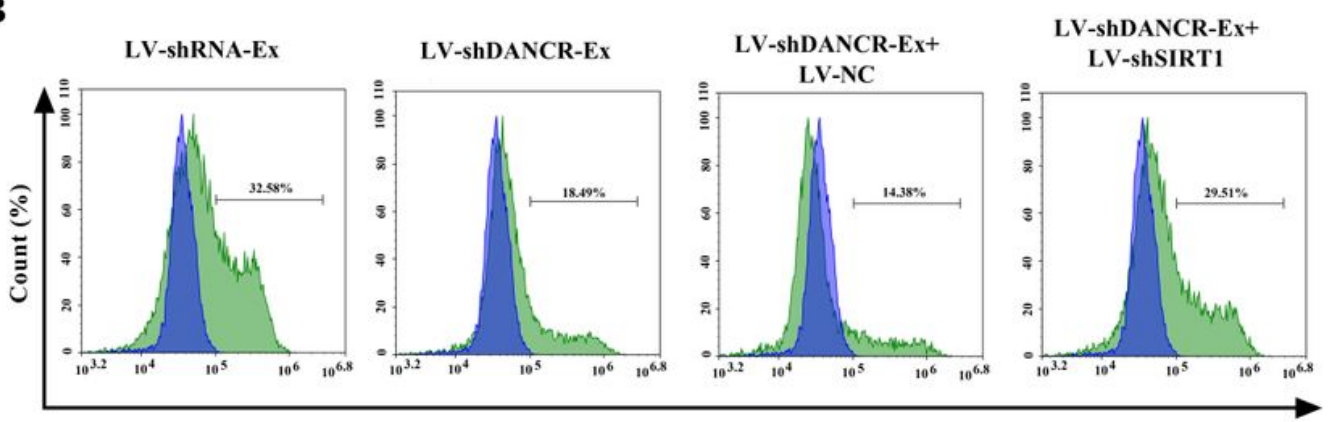

FOXP3

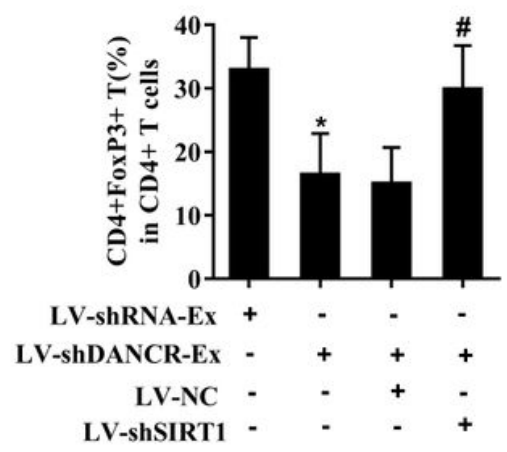

Figure 7

After interference with DANCR, the collected BMMSC-Ex inhibited Treg cell differentiation by up-regulating SIRT1 expression in CD4+ T cells. BMMSCs were isolated and transfected with LV-shDANCR or LV-shRNA, and the BMMSC-Ex was collected and co-cultured with CD4+ T cells (transfected with LV-NC or LVshSIRT1). The concentration of added exosomes was $50 \mathrm{ng} / \mathrm{mL}$ and cells were induced for Treg cell differentiation. (A) The protein level of SIRT1 was detected by Western blot. (B) The proportion of Treg cells was determined by flow cytometry. (C-D) The expression of Foxp3 and IL-10 in CD4+ T cells was detected by qRT-PCR. * $P<0.05$, ** $P<0.01$ versus the LV-shRNA-Ex group; \# $P<0.05$, \#\# $P<0.01$ versus the LV-shDANCR-Ex + LV-NC group. 
A

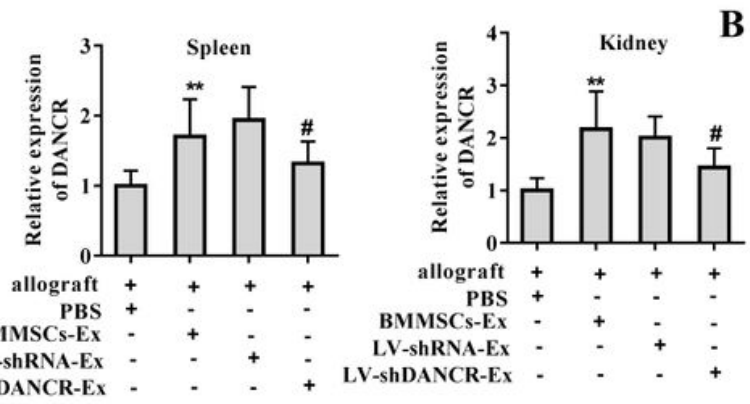

B

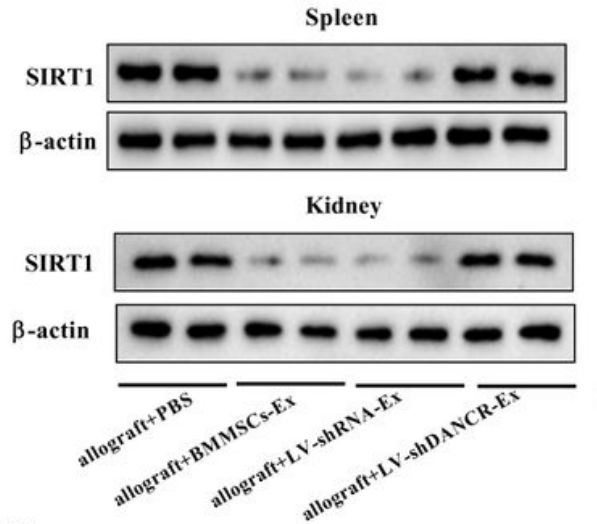

E

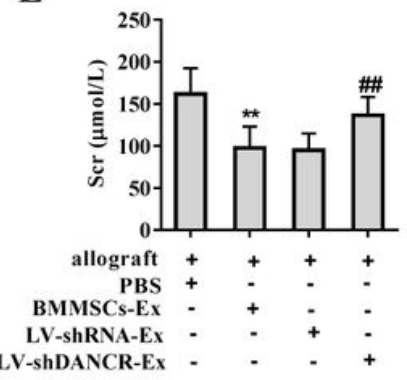

LV-ShDANCR-Ex
C

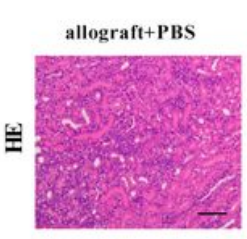

D

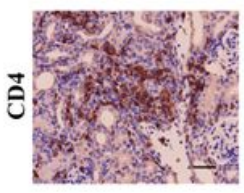

G

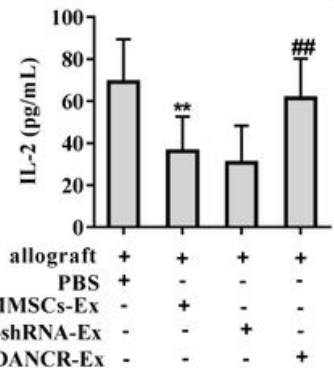

H
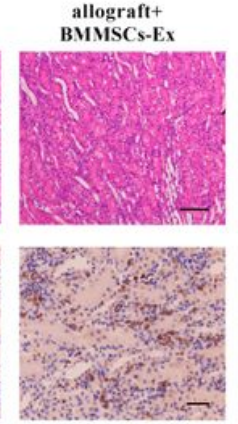
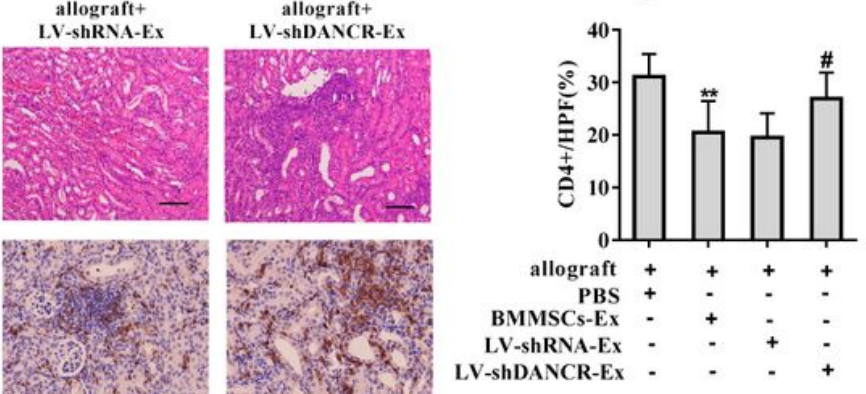

F

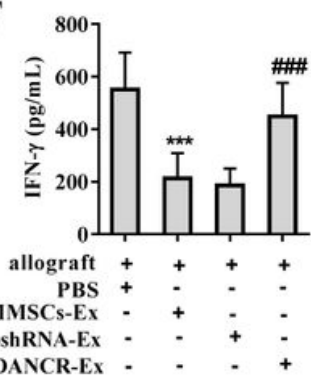

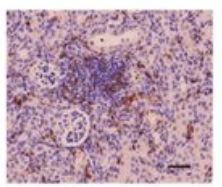

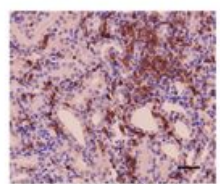

LV-shDANCR-Ex
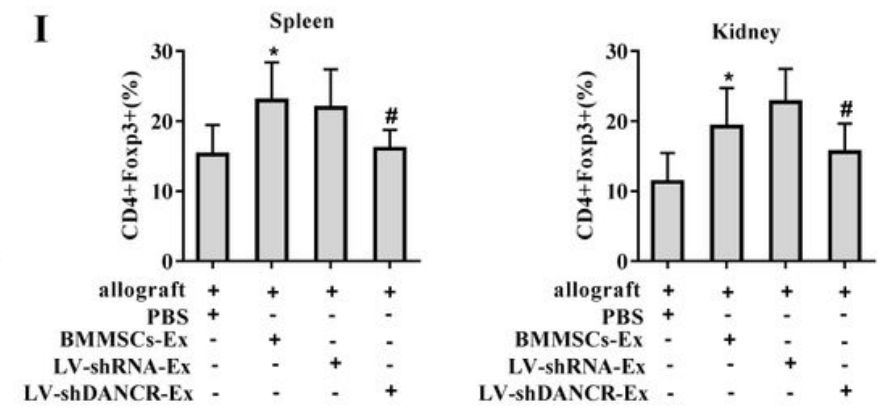

\section{Figure 8}

After interference with DANCR, the collected BMMSC-Ex aggravated kidney transplant rejection in mice. The model of kidney transplantation in mice was established. BMMSCs were transfected with LV-shRNA, and exosomes of BMMSCs were collected (LV-shRNA-Ex). BMMSCs were transfected with LV-shDANCR, and exosomes of BMMSCs were collected (LV-shDANCR-Ex). Mice were grouped ( $n=7$ per group): the allograft + PBS group (mice were infused with PBS through caudal vein 1 day before and 1 day after kidney transplantation), the allograft + BMMSC-Ex group (mice were infused with BMMSC-Ex through caudal vein 1 day before and 1 day after kidney transplantation), the allograft + LV-shRNA-Ex group (mice were infused with LV-shRNA-Ex through caudal vein 1 day before and 1 day after kidney transplantation), the allograft + LV-shDANCR-Ex group (mice were infused with LV-shDANCR-Ex through caudal vein 1 day before and 1 day after kidney transplantation). Six days after transplantation, the recipient mice were sacrificed, the spleen tissues, transplanted kidney tissues, serum, and plasma samples were collected for analysis. (A) QRT-PCR was used to detect the expression of DANCR in the spleen and transplanted kidney. (B) Western blot was used to detect SIRT1 expression in the spleen and kidney. (C) The inflammation of kidney tissues was observed by HE staining. Scale bar: $100 \mu \mathrm{m}$. (D) The infiltration of CD4+ T cells in 
kidney tissues was observed by CD4 immunohistochemistry. Scale bar: $40 \mu \mathrm{m}$. (E) SCr levels were measured by an automatic biochemical analyzer. (F-H) The expression of rejection-related factors (IFN- $-\gamma$, IL-2, and IL-17) in plasma was detected by ELISA. (I) The proportion of Treg cells in the spleen and kidney was determined by flow cytometry. ${ }^{*} P<0.05,{ }^{*} P<0.01$, ${ }^{* \star *} P<0.001$ versus the allograft + PBS group; \# $P<0.05$, \#\# $\mathrm{P}<0.01$, \#\#\# $\mathrm{P}<0.001$ versus the allograft + LV-shRNA-Ex group. 